\title{
Photocoagulation for peripheral neovascularisation in diabetes
}

\author{
D V INGLESBY, G S TURNER, W E SCHULENBURG, AND E M KOHNER \\ From the Department of Medicine, Hammersmith Hospital, Du Cane Road, London W12 OHS
}

SUMMARY A study of 90 eyes in 72 diabetic patients with peripheral retinal neovascularisation treated by photocoagulation indicated that the subsequent development of further peripheral or disc new vessels is infrequent if panretinal photocoagulation is the initial treatment procedure. Focal or sectorial treatment may be considered only if a small area of neovascularisation is present initially and provided regular follow-up can be guaranteed.

New vessel proliferation from the optic disc, the retinal surface, or the surface of the iris occurs as a complication of diabetes and is presumed to be a response to widespread retinal ischaemia. The treatment of disc new vessels (NVD) has now been rationalised as the result of many studies, the most impressive of which have been the Diabetic Retinopathy Study (DRS) in the United States, ${ }^{\prime}$ and the British Multicentre Study. ${ }^{2}$ The progress of iris neovascularisation can be arrested or even reversed if fibrotic changes are absent. ${ }^{3}$ In both of these conditions the most effective treatment is panretinal photocoagulation (PRP), applied either by means of the xenon arc or the argon laser. The effect of each on NVD is similar, though the use of argon is accompanied by fewer side effects. ${ }^{45}$

The best form of management of peripheral retinal neovascularisation (new vessels elsewhere; NVE) is Correspondence to Dr D V Inglesby, Moorfields Eye Hospital, City Road, London EC1V 2PD. undecided. The presence of such vessels constitutes a threat to vision because of the potential for haemorrhage, though the risk is not of the same order as that posed by NVD. However, if NVE are accompanied by preretinal or vitreous haemorrhage, the eye enters the 'high risk' category of the DRS. ${ }^{4}$ This fact has caused many ophthalmologists to treat eyes with NVE before high risk characteristics become manifest. The generally accepted treatment consists of focal or sectorial photocoagulation, with PRP being employed for those cases with widespread NVE. ${ }^{6}$ The original policy of this unit, to employ focal treatment only, was influenced by the work of Okun et al. ${ }^{7}$ However, the clinical impression gained in the unit over 15 years of treating such eyes is that those receiving focal or sectorial treatment progress to develop further new vessels with such regularity that PRP is now usually undertaken as the initial procedure, despite there having been no formal change in policy (Fig. 1).

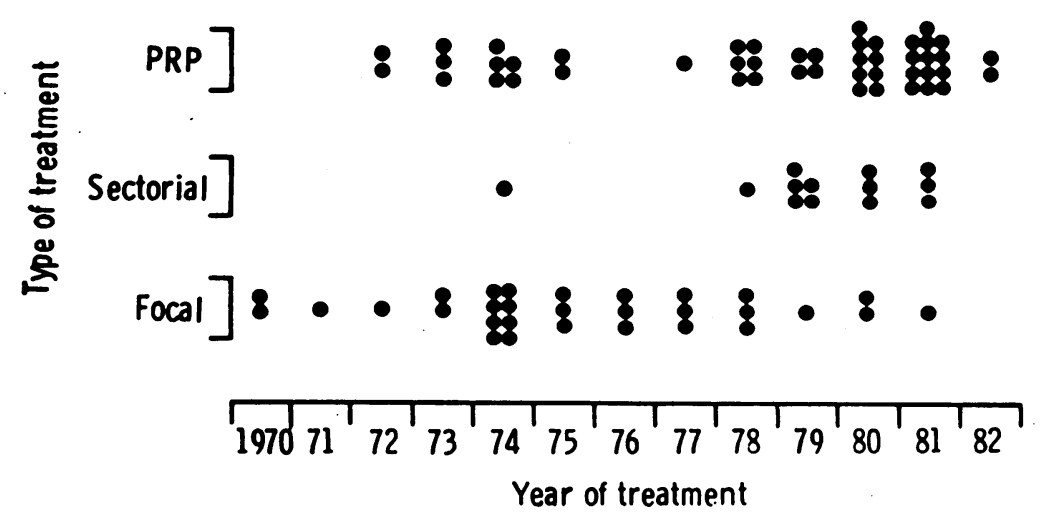

Fig. 1 Distribution of types of treatment, 1970-82. (PRP=panretinal photocoagulation). 
The present study was performed to test this clinical impression - that is, to determine what proportion of eyes in each of the three treatment groups (focal, sectorial, PRP) proceeded to further neovascularisation after the initial treatment, and whether this progression was related to the extent of new vessel formation at that time.

\section{Patients and methods}

All the patients were attending the Diabetic Retinopathy Clinic at the Hammersmith Hospital and were treated between 1970 and 1982 . The notes of all patients treated for NVE were inspected. Seventytwo patients were included in the study, of whom 25 were female, accounting for 30 of the 90 eyes studied. Fifty-eight of the patients were being treated with insulin, the remaining 14 being controlled on oral hypoglycaemic agents. The age at onset of diabetes ranged from 2 to 61 years (mean 27 years). The mean duration of known diabetes at the time of diagnosis of NVE was 15 years (Table 1). The mean age at diagnosis of NVE was 42 years (range 22-64 years). None of the eyes in the study had received photocoagulation for any reason prior to the NVE being treated, nor had any of the patients undergone pituitary ablation.

NVE were defined as those new vessels arising from areas of the fundus more than one disc diameter away from the optic disc. All patients had colour photography of the eyes, and usually but not invariably fluorescein angiography, performed prior to treatment. Included in the study were those displaying NVE in these pictures as well as those who were recorded in the notes (in the opinion of a consultant ophthalmologist) as having had NVE outside the field of the standard retinal photographs as defined in the Hammersmith Hospital Grading System. ${ }^{8}$ In none of the eyes were NVD present at the time of the initial treatment, nor was there more than a trace of fibrous retinitis proliferans in any eye. Excluded from the study were those eyes displaying fluorescein leakage from the disc even if NVD were not recorded

Table 1 Age of onset and duration of diabetes at time of diagnosis of $N V E$ in individual eyes

\begin{tabular}{lcclc}
\hline $\begin{array}{l}\text { Known duration of } \\
\text { diabetes at diagnosis }\end{array}$ & \multicolumn{2}{l}{ Age at onset of diabetes (years) } & Total \\
\cline { 2 - 5 } of NVE (years) & $0-29$ & $30-59$ & $\geqslant 60$ & \\
\hline $0-5$ & 0 & 12 & 1 & 13 \\
$6-10$ & 1 & 3 & 0 & 4 \\
$11-15$ & 15 & 7 & 0 & 22 \\
$16-20$ & 15 & 10 & 0 & 25 \\
$21-30$ & 16 & 6 & 0 & 22 \\
$>30$ & 4 & 0 & 0 & 4 \\
Total & 51 & 38 & 1 & 90 \\
\hline
\end{tabular}

in the notes or observed on the colour photographs. Excluded also were those eyes thought to display grade 1 NVE but which did not show the characteristic leakage of fluorescein; such lesions would today be considered to be intraretinal microvascular abnormalities.

\section{TREATMENT}

Focal photocoagulation treatment implies that given to the retina underlying the area of NVE, with a rim of adjacent retina being incorporated. Sectorial treatment involves in addition the creation of burns in a sector of the more peripheral retina, generally about an arc of $60^{\circ}$, with the site of NVE being within the apex of the sector. PRP implies clusters of burns around the foci of NVE with widespread scattered treatment in the remainder of the retinal periphery. Obviously the density of such treatment may vary. An arbitrary distinction was made here between 'light' and 'dense' PRP, the former being fewer than 2400 argon laser burns of $500 \mu \mathrm{m}$ size, or fewer than 200 xenon burns $4.5^{\circ}$ in size. Forty-seven eyes received PRP, 13 received sectorial treatment, and 30 had focal treatment. Fifty-six of the eyes were treated with the argon laser.

After treatment all patients were followed up for at least two years, the maximum being 13 years, mean five years. During this time examinations were regularly carried out, at least three times annually. Colour photographs were taken at least annually. Further neovascularisation was therefore detected and treated promptly when it occurred, PRP generally being applied on these occasions.

Statistical methods. The significance of the findings was assessed by the $\chi^{2}$ method.

\section{Results}

Of the 90 treated eyes 31 (34\%) developed subsequent neovascularisation (NVD in seven cases, NVE in 24 cases). The mean duration from treatment to NVD formation was two years, (range one month to seven years), and that from treatment to further NVE appearance was 2.5 years (range nine months to eight years). Seven of 47 eyes receiving PRP developed further new vessels, compared with $6 / 13$ receiving sectorial, and $18 / 30$ receiving focal treatment. Of the seven which had received PRP four had 'light' PRP (mean 1800 argon burns). A further eye receiving only 1000 argon burns later developed rubeosis iridis and then received further treatment. These results showed a significant $(p<0.001)$ difference in favour of PRP over focal triatment, and a less significant $(0.01<p<0.02)$ advantage over sectorial treatment in preventing further neovascularisation (Fig. 2). 
$\square$ No further new vessels

Q NVE

NVD

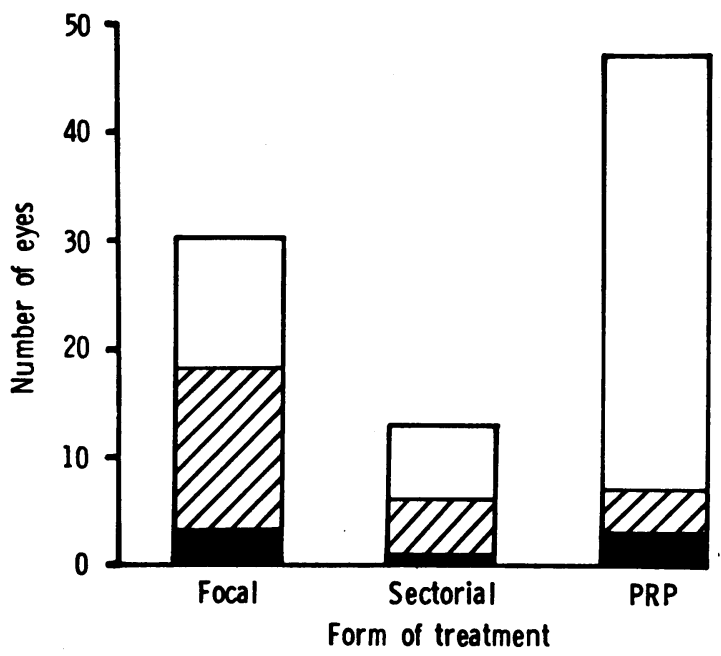

Fig. 2 Subsequent development of new vessels in eyes grouped according to type of initial treatment. (PRP= panretinal photocoagulation).
The histograms in Fig. 3 illustrate the eyes in four groups corresponding to the number of retinal quadrants containing NVE at the time of the initial treatment, and show the proportion of eyes which developed NVD or NVE for each mode of treatment in each group. It can be appreciated that in each of the four groups PRP was associated with a smaller incidence of further new vessel formation than were the other forms of treatment, but that the numbers involved were too small to apply the $\chi^{2}$ test.

The proportion of eyes in each group that received argon or xenon treatment is demonstrated in Fig. 4, showing those eyes that developed further new vessels in addition to the group as a whole. It can be seen that there was no difference in this series between argon and xenon treatment in terms of the likelihood of subsequent new vessel formation.

The best corrected visual acuity before treatment was generally better than 6/12. Only 14 eyes were between $6 / 12$ and $6 / 60$. After treatment, and apart from temporary changes, there was loss of two lines (Snellen) in three eyes and loss of one line in eight eyes. Two eyes impro d by two lines, six by one line; $71 / 90$ remained the same. No record was made of visual field changes.

\section{Discussion}

Oosterhuis et al." state that 'in patients with peripheral new vessel formation photocoagulation

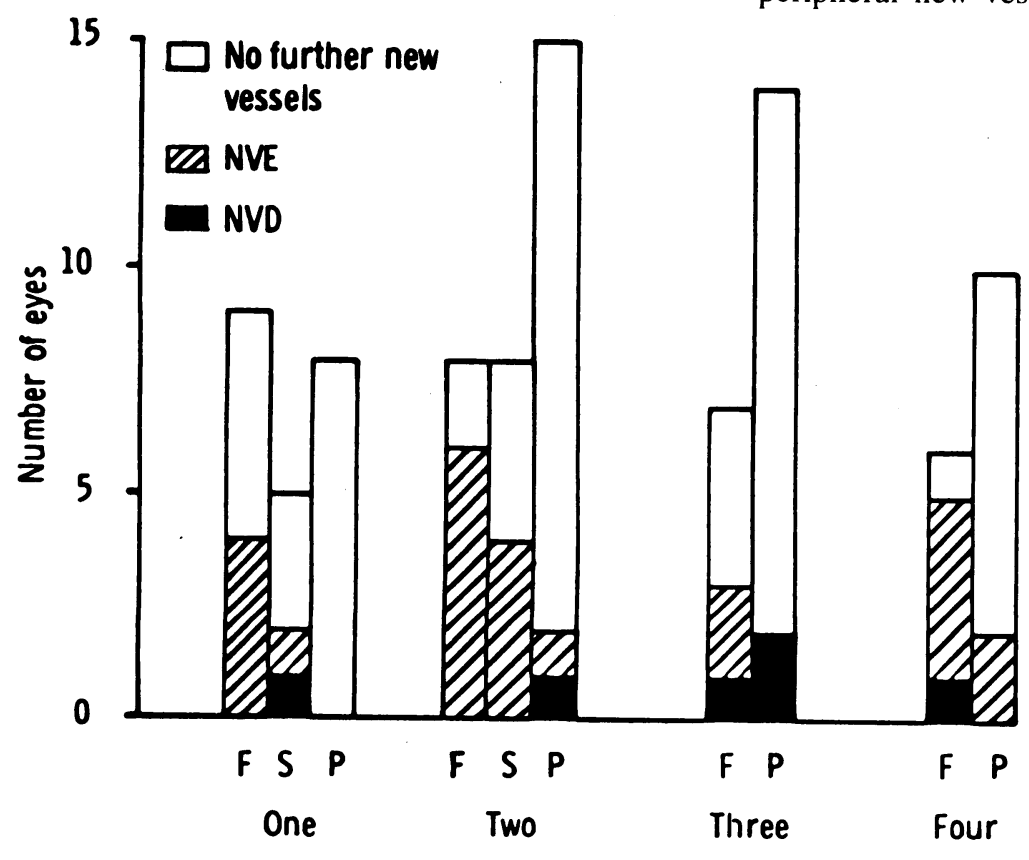

Fig. 3 Subsequent development of new vessels in eyes grouped according to number of retinal quadrants initially containing $N V E$, and type of treatment received. ( $F=$ focal; $S=$ sectorial; $P=$ panretinal photocoagulation). 


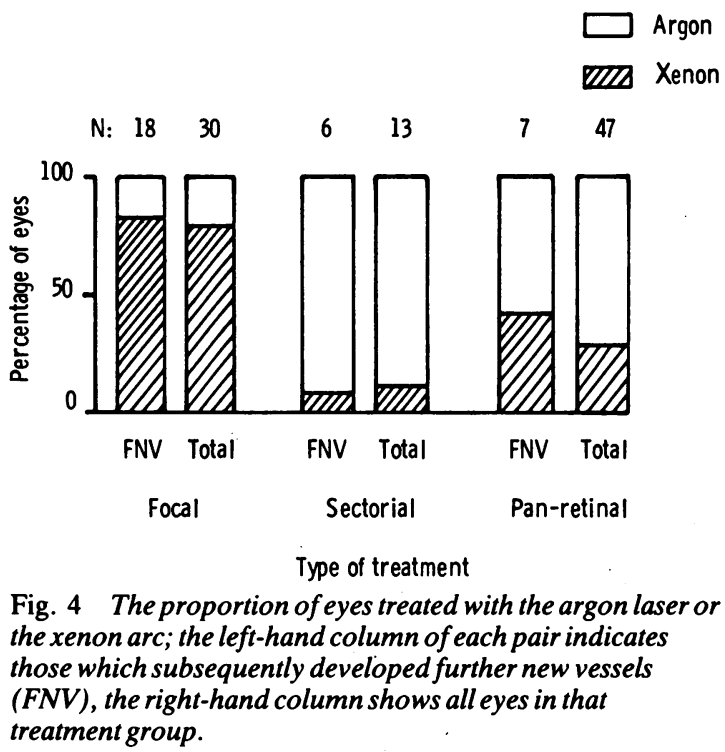

must be performed before disasterous complicating lesions such as vitreous haemorrhage, glial strands, or signs of shrinkage have developed.' The DRS, in referring to eyes with proliferative retihopathy but without high risk characteristics, asks if the extensive scatter treatment used in the study was necessary, whether less extensive treatment might suffice, or whether in fact it might be safe or even wise to defer treatment. ${ }^{1}$ We feel, like Oosterhuis et al., that treatment is necessary because the presence of such lesions is a definite risk to vision, although in a small proportion of cases the abnormal vessels may regress spontaneously, ${ }^{10}$ as do $4 \%$ of NVD. ${ }^{4}$ Eyes with moderate or severe NVE, if associated with vitreous or preretinal haemorrhage (i.e., in the high risk group) develop vision of less than $5 / 200$ in $36.8 \%$ cases over a two-year period," and eyes with any grade of NVE have a definite and increased risk of developing high risk characteristics if left untreated. ${ }^{4}$ This finding has recently been confirmed in the report of the British Multicentre Study, ${ }^{2}$ which showed that $50 \%$ of untreated eyes containing NVE developed NVD within five years. Also to be remembered is the fact that, even with treatment, eyes with high risk features still have an appreciable chance of going blind $(12-15 \%)$ in five years. ${ }^{12}$ However, as the authors of these figures are careful to point out, they include some eyes that were diagnosed and treated at a more advanced stage than would be the case with regular surveillance of cases progressing from the milder stages of disease.

Against this background many ophthalmologists advocate the tretment of NVE, but there are no reports concerning this matter specifically, and there is no consensus as to the most appropriate method. The presence of NVE indicates retinal ischaemia, and from fluorescein angiography it is usually apparent that ischaemia is widespread even if only one or two small foci of NVE are present. Therefore focal treatment, although removing the immediate source of risk to the eye, will not remove the continuing stimulus to further neovascularisation. Not surprisingly, therefore, the present study shows that, overall, PRP is more effective than less extensive treatment in preventing subsequent neovascularisation. This is in agreement with the British Multicentre Study, which found that 12 eyes treated for NVE and which later developed NVD had had less treatment than the 30 which did not progress in this way. ${ }^{2}$

One criticism of the data presented here is that the group receiving focal treatment has been followed up for a longer period than those receiving PRP or sectorial treatment (Fig. 1). However, we have shown that the mean duration between treatment and the development of further new vessels is from two to 2.5 years, and all eyes have been followed for at least two years. In addition Oosterhuis $e t$ al. found that in the latter three years of their five-year posttreatment follow-up most of the eyes were in a relatively stable situation, so we believe that no great error has been incurred by our comparison of the treatment groups.

It is to be remembered that even in capable hands the application of PRP with the argon laser imparts a small risk to central vision, and peripheral field and night vision are slightly impaired. However, we consider that PRP is the appropriate form of treatment as the initial procedure for most eyes displaying NVE. Limited treatment should be reserved only for those few eyes with very early NVE, without widespread ischaemia, in patients who can be thoroughly examined regularly.

The authors acknowledge the work of the ophthalmologists and photographers who worked in the unit during the period of management of the patients in the study.

This work was supported by a group grant from the Scientific Section of the British Diabetic Association.

\section{References}

1 Diabetic Retinopathy Study Research Group. Photocoagulation treatment of proliferative diabetic retinopathy: clinical applications of DRS findings. Report number 8. Ophthalmology 1981; 88: $583-600$.

2 British Multicentre Study Group. Photocoagulation for proliferative diabetic retinopathy: a randomised controlled clinical trial using the xenon-arc. Diabetologia 1984; 26: 109-15.

3 Little HL, Rosenthal AR, Dellaporta A, Jacobson DR. The effect of pan-retinal photocoagulation on rubeosis iridis. Am J Ophthalmol 1976; 81: 804-9.

4 Diabetic Retinopathy Study Research Group. Photocoagulation treatment of proliferative diabetic retinopathy: the second report of DRS findings. Ophthalmology 1978; 85: 82-106. 
5 Plumb AP, Swan AV, Chignell AM, Shilling JS. A comparative trial of xenon-arc and argon laser in the treatment of proliferative diabetic retinopathy. Br J Ophthalmol 1982; 66: 213-8.

6 Morse PH. Rationale for the treatment of diabetic retinopathy. Trans Ophthalmol Soc UK 1976; 96: 86-9.

7 Okun E, Johnston GP, Boniuk I. Management of diabetic retinopathy: a stereoscopic presentation. St Louis: Mosby, 1971: chapter 5 .

8 Oakley NW, Hill DW, Joplin GF, Kohner EM, Fraser TR. Diabetic retinopathy. 1 . The assessment of severity and progress by comparison with a set of standard fundus photographs. Diabetologia 1967; 3: 402-5.

9 Oosterhuis JA, Beintema MR, Lemkes HHLM, Nikkels R, Terpstra J. Photocoagulation treatment in diabetic retinopathy: a two-year pre- and five-year post-treatment study. Doc Ophthalmol 1979; 48: 101-62.

10 Dobree JH. Proliferative diabetic retinopathy: evolution of the retinal lesions. Br J Ophthalmol 1964; 48: 637-49.

11 Diabetic Retinopathy Study Research Group. Preliminary report on effects of photocoagulation therapy. Am J Ophthalmol 1976; 81: 383-96. 